\title{
Utilization of Green Formulation Technique and Efficacy Estimation on Cell Line Studies For Dual Anticancer Drug Therapy With Niosomes
}

\section{Khan, Daulat Haleem}

2019-12-15

Khan, D H , Bashir , S , Correia , A, Khan , M I, Figueiredo , P , Santos , H A \& Peltonen, L 2019 , ' Utilization of Green Formulation Technique and Efficacy Estimation on Cell Line Studies For Dual Anticancer Drug Therapy With Niosomes ' , International Journal of Pharmaceutics , vol. 572 , 118764 . https://doi.org/10.1016/j.ijpharm.2019.118764

http://hdl.handle.net/10138/320373

https://doi.org/10.1016/j.jpharm.2019.118764

cc_by_nc_nd

acceptedVersion

Downloaded from Helda, University of Helsinki institutional repository.

This is an electronic reprint of the original article.

This reprint may differ from the original in pagination and typographic detail.

Please cite the original version. 
Utilization of green formulation technique and efficacy estimation on cell line studies for dual anticancer drug therapy with niosomes

Daulat Haleem Khan ${ }^{1,2,3}$, Sajid Bashir ${ }^{1}$, Alexandra Correia ${ }^{2}$, Muhammad Imran Khan ${ }^{4}$

Patrícia Figueiredo $^{2}$, Hélder A. Santos ${ }^{2,5}$, Leena Peltonen ${ }^{2}$.

${ }^{1}$ College of Pharmacy, University of Sargodha, Sargodha, Pakistan

${ }^{2}$ Drug Research Program, Division of Pharmaceutical Chemistry and Technology, Faculty of Pharmacy, FI-00014, University of Helsinki, Finland

${ }^{3}$ Lahore College of Pharmaceutical Sciences, 54000 Lahore, Pakistan

${ }^{4}$ Riphah Institute of Pharmaceutical Sciences, Riphah International University, 54000

Lahore, Pakistan

${ }^{5}$ Helsinki Institute of Life Science (HiLIFE), FI-00014, University of Helsinki, Finland

*Corresponding author

Contact information for corresponding author:

Leena Peltonen: Drug Research Program, Division of Pharmaceutical Chemistry and

Technology, Faculty of Pharmacy, P.O. Box 56, Viikinkaari 5 E, FI- 00014 University of Helsinki, Finland.

E-mail address: leena.peltonen@helsinki.fi

Phone $+358-504480726$.

Declarations of interest: None 


\begin{abstract}
The aim of the present study was to prepare niosome formulations for the simultaneous encapsulation, dual drug therapy, of two anticancer drugs by the ecological probe sonication method. Poloxamer and sorbitan monostearate were used as surface active agents in niosomes, and the water soluble doxorubicin and poorly-water soluble paclitaxel were used as anticancer drugs. Thorough physicochemical analysis were performed for the niosomes, and their cytotoxicity and activity were evaluated on MCF-7 and PC3-MM2 cancer cell lines. Prepared niosomes were small in size with sizes ranging from $137 \mathrm{~nm}$ to $893 \mathrm{~nm}$, and entrapment efficiencies were high, ranging from $91.24 \%$ to $99.99 \%$. During the four weeks stability testing, the particle size remained stable. The niosomal formulations showed in vitro sustained drug release profiles for doxorubicin and clearly increased the dissolution rate of poorly water soluble paclitaxel. The incorporation of both the drugs into niosomes improved cell penetration and antiproliferative activity of the drugs PC3-MM2 cell lines. As a conclusion, doxorubicin and paclitaxel loaded niosome formulations resulted in relatively stable, small sized niosomes with improved drug release profiles, low toxicity, better cell penetration and antiproliferative activity. The niosomes showed synergistic effect due to the presence of both drugs, which can overcome multidrug resistance.
\end{abstract}

Keywords: antiproliferative activity, cell penetration, doxorubicin, dual drug therapy, paclitaxel, niosomes 


\section{Introduction}

Cancer is one of the causative factors of death around the world in many countries (Yingchoncharoen et al., 2016), and chemotherapy is among the approaches, which is effective against multiple cancers (Tahir et al., 2017). However, the chemotherapeutics have toxic adverse effects on healthy living cells regardless of selectivity (Zheng et al., 2015). Due to high toxicity and shorter half-life, the use of chemotherapeutics is limited to avoid toxicity. Accordingly, in order to avoid the hazardous adverse effects, the challenge is that chemotherapeutical agents are needed to be delivered only to the cancer cells with minimum delivery to normal cells (Yingchoncharoen et al., 2016; Tahir et al., 2017).

Different nanosized systems are the carrier of choice for the efficient loading of the drugs (hydrophilic or hydrophobic) in order to reach site specificity, prolonged circulation time in body, and lower toxicity (Sharma et al., 2015). Niosomes, vesicular structures composed of non-ionic surfactants, are capable of encapsulating both hydrophobic as well as hydrophilic drugs (Sharma et al., 2016). Niosomes have advantages over liposomes, like higher stability and entrapment efficiency, biocompatibility, non-immunogenicity and lower costs (Manconi et al., 2002; Tavano et al., 2014). A number of non-ionic surfactants, like alkyl ethers, alkyl esters, polysorbates, poloxamers and alkyl amides, have been used to produce niosomes (Di Marzio et al., 2011; Escudero et al., 2014; Moghassemi et al., 2014).

Poloxamers are promising non-ionic polymeric surfactants, which have been used for niosome production and delivery of anticancer drugs for the treatment of multidrug resistant cancers. Pluronic L121 is one of the poloxamers used for the encapsulation of cytotoxic drugs, and it is also P-glycoprotein (P-gp) inhibitor (Yang et al., 2007b). In most of the niosomal studies, only a single surfactant has been used (Di Marzio et al., 2011; 
Escudero et al., 2014; Moghassemi et al., 2014). However, when two or more non-ionic amphiphiles are used, more stable, small in size, monodispersed niosomes with better drug release profiles can be reached (Khan et al., 2016).

Niosomes are produced by different methods, the most adopted being reverse phase evaporation, thin film hydration, ether injection and shaking methods (Kanaani et al., 2017; Ravalika and Sailaja, 2017). These methods are time consuming and expensive, they use organic solvents, and after the production, solvent removal is laborous. Probe sonication method has been developed to overcome these problems (Dufes et al., 2000). It is a green technique with low energy consumption and without the addition of any organic solvents. In this method, only aqueous drug phase is mixed with surfactants, cholesterol and other bilayer membrane additives (Khan et al., 2017).

Doxorubicin (DOX) and paclitaxel (PXT), chemotherapeutic agents belonging to anthracycline and taxanes classes of cytotoxic drugs, respectively, are effective against number of cancers, including breast and prostatic cancers (Kim et al., 2015; Pawar et al., 2016). DOX is hydrophilic in nature, but, PXT is hydrophobic, which limits its bioavailability (Alemi et al., 2018; Behnam et al., 2018). Besides, the delivery of these cytotoxic drugs is challenging due to their highly toxic adverse effects and drug resistance (Liu et al., 2017; Teixeira et al., 2017; Sayed et al., 2018). However, it has been shown that the combination of DOX and PXT have a great attraction due to synergistic effect with reduced systematic toxicity and higher antitumor efficacy (Devita et al., 1975; Chabner and Roberts, 2005; Al-Lazikani et al., 2012; Ag Seleci et al., 2017; Alemi et al., 2018, Yang et al., 2019).

In the present study, co-delivery formulation of two anticancer drugs, DOX and PTX were prepared by an environmental friendly probe sonication method. The aims of the dual drug 
therapy were synergistic effects with lower toxicity levels and higher antitumor efficacy. Niosomes containing only a single drug, as well as both the drugs, were formulated. Pluronic L121 and Span 60 surfactants were utilized for production of niosomes. Performance of niosomal formulations was confirmed by thorough physicochemical analysis and efficiency of the formulations were confirmed in vitro in different cancer cell lines.

\section{Materials and methods}

\subsection{Materials}

Doxorubicin hydrochloride (Fluorochem, UK) and paclitaxel (Fluorochem, UK) were studied chemotherapeutic drug substances. Sorbitan Monostearate (Span 60, SigmaAldrich, USA) and Polyethylene oxide - Polypropylene oxide - Polyethylene oxide (PEOPPO-PEO) block copolymer (Pluronic L121, Mn 4400, Sigma-Aldrich, USA) were used as bilayer membrane formers in niosomes. Cholesterol (Sigma-Aldrich, USA) was used as membrane stabilizer and dicetylphosphate (DCP, Sigma-Aldrich, USA) as charge imparting agent. Hank's Balanced Salt Solution (HBSS), and Dulbecco's Modified Eagle's Medium (DMEM) were purchased from HyClone (USA). Tween 80 was used as a solubilizing agent in dissolution testing (Sigma-Aldrich, USA). Water used in all the tests was Milli-Q water (Millipore, Merckmillipore, USA).

\subsection{Methods}

\subsubsection{Preparation of niosomes}

The niosomes were prepared by probe sonication method (Khan et al., 2019). First, the drugs, doxorubicin- $\mathrm{HCl}(\mathrm{DOX})$ and paclitaxel (PXT), individually or as a combination, were mixed with $15 \mathrm{~mL}$ of water with the aid of magnetic stirrer, after which cholesterol, 
Span 60, Pluronic L121, and dicetylphosphate (DCP) were added. The compositions of different studied formulations are indicated in Table 1. The mixtures were then subjected to probe sonication (Vibra Cell, Sonics \& Materials, Inc., USA) for 5 min time at $57^{\circ} \mathrm{C}$ probe temperature in a pulsatile manner (50 sec sonication with $10 \mathrm{sec}$ pause) at an amplitude of $30 \%$. After probe sonication, niosome formulations were collected and stored at $4^{\circ} \mathrm{C}$ for further characterization and cell line studies. 
Table 1: Compositions of studied niosome formulations.

\begin{tabular}{cccccccc}
\hline Formulations & $\begin{array}{c}\text { Span 60 } \\
(\mathbf{m g})\end{array}$ & $\begin{array}{c}\text { Pluronic } \\
\text { L121 (mg) }\end{array}$ & $\begin{array}{c}\text { Cholesterol } \\
(\mathbf{m g})\end{array}$ & $\begin{array}{c}\text { DCP } \\
(\mathbf{m g})\end{array}$ & $\begin{array}{c}\text { DOX } \\
(\mathbf{m g})\end{array}$ & $\begin{array}{c}\text { PXT } \\
(\mathbf{m g})\end{array}$ & $\begin{array}{c}\text { Water } \\
(\mathbf{m L})\end{array}$ \\
\hline D1 & 43 & 290 & 77.3 & 1 & 2 & - & 15 \\
D2 & 43 & 290 & 77.3 & 2 & 2 & - & 15 \\
D3 & 43 & 290 & 77.3 & 0 & 2 & - & 15 \\
D4 & 43 & 246 & 77.3 & 1 & 2 & - & 15 \\
D5 & 43 & 334 & 77.3 & 1 & 2 & - & 15 \\
P1 & 43 & 290 & 77.3 & 1 & - & 2 & 15 \\
P2 & 43 & 290 & 77.3 & 2 & - & 2 & 15 \\
P3 & 43 & 290 & 77.3 & 0 & - & 2 & 15 \\
P4 & 43 & 246 & 77.3 & 1 & - & 2 & 15 \\
P5 & 43 & 334 & 77.3 & 1 & - & 2 & 15 \\
DP1 & 43 & 290 & 77.3 & 1 & 2 & 2 & 15 \\
DP2 & 43 & 290 & 77.3 & 2 & 2 & 2 & 15 \\
DP3 & 43 & 290 & 77.3 & 0 & 2 & 2 & 15 \\
DP4 & 43 & 246 & 77.3 & 1 & 2 & 2 & 15 \\
DP5 & 43 & 334 & 77.3 & 1 & 2 & 2 & 15 \\
E1 & 43 & 290 & 77.3 & 1 & - & - & 15 \\
E2 & 43 & 290 & 77.3 & 2 & - & - & 15 \\
E3 & 43 & 290 & 77.3 & 0 & - & - & 15 \\
E4 & 43 & 246 & 77.3 & 1 & - & - & 15 \\
E5 & 43 & 334 & 77.3 & 1 & - & - & 15 \\
\hline
\end{tabular}

2.2.2 Particle size, size deviation and zeta-potential

The diameter of the niosomes (z-average) and polydispersity index (PDI), based on dynamic light scattering (DLS) technique, as well as zeta-potential, were measured by using Zeta-sizer Nano ZS (Malvern Instruments Ltd., USA). The niosomal dispersions were diluted with water before the measurement to avoid multi scattering phenomenon. All the measurements were performed in triplicate.

\subsubsection{Drug entrapment efficiency}

For drug entrapment efficiency determinations, the formulations were ultracentrifuged at 14,500 rpm for $45 \mathrm{~min}$ time (Sigma Laborzentrifugen, D-37520, Germany). The supernatant was collected, the pellet at the bottom of the centrifuge tube was washed twice with water, water was collected, and centrifugation was repeated. Drug concentration in the aqueous portion of supernatants was determined. For PXT determination, high performance 
liquid chromatography (HPLC, Agilent 1260, Agilent Technologies, USA), and for DOX, spectrophotometric analysis (Varioskan Flesh, Thermo Fisher Scientific Inc., USA), were used. The percentage entrapment (EE\%) of drugs were calculated according to the following equation (Equation 1) (Li et al., 2016; Maestrelli et al., 2017):

$\mathrm{EE} \%=\left[\left(\mathrm{Q}_{\mathrm{t}}-\mathrm{Q}_{\mathrm{r}}\right) / \mathrm{Q}_{\mathrm{t}}\right] \times 100$,

where $\mathrm{Q}_{t}$ is the amount of drug initially used for the preparation of niosomes and $\mathrm{Q}_{\mathrm{r}}$ is the amount of drug present in supernatant after centrifugation.

\subsubsection{Transmission electron microscopy}

The morphology of the niosomes was investigated by the transmission electron microscopy (TEM, Jeol JEM-1400, Jeol Ltd, Japan). For TEM analysis, small amount of niosomal dispersions were inserted on a carbon coated 200-mesh sized copper grid. The mesh was positioned horizontally for one minute, superfluous was removed with the aid of filter paper and one drop of $2 \%$ uranyl acetate was placed on the sample for staining (Somjid et al., 2018).

2.2.5 Attenuated total reflectance - fourier transform infrared (ATR-FTIR) spectroscopy

The possible interactions between the drug, the non-ionic surfactants and other membrane additives were studied by attenuated total reflectance - fourier transform infrared (ATRFTIR) spectroscopy. The ATR-FTIR spectra of all the individual components, their physical mixtures and niosome formulations containing DOX, PXT and DOX+PXT were measured. For ATR-FTIR analysis, the niosome dispersions were centrifuged, and the dried pellet was analyzed. The spectra were recorded by using FTIR spectrophotometer (Bruker Optics, Germany) with an additional horizontal accessory of ATR (MIRacle, Pike 
Technology, Inc., Germany). The spectra were recorded at an ambient temperature between wavenumbers of $400-4500 \mathrm{~cm}^{-1}$ with $4 \mathrm{~cm}^{-1}$ resolution by using OPUS 5.5 software.

\subsubsection{Thermal analysis}

The physical states of the DOX and PXT in the formulations were estimated by using differential scanning calorimetry (DSC 823 ${ }^{\mathrm{e}}$, Mettler Toledo, USA). Pure DOX, PXT, individual niosome constituents, their physical mixtures, and formulations containing DOX, PXT and DOX+PXT were accurately weighed (3-5 mg) in closed aluminum pan. For DSC analysis, the niosome dispersions were centrifuged, and the dried pellet was analyzed. The thermal scanning was carried out from $25^{\circ} \mathrm{C}$ to $260^{\circ} \mathrm{C}$ with a heating rate of $5^{\circ} \mathrm{C} / \mathrm{min}$. The analysis were conducted under the nitrogen gas flow $(50 \mathrm{ml} / \mathrm{min})$.

\subsubsection{Stability studies}

Four weeks stability study of niosome formulations was performed by storing the niosomal dispersions in sealed $20 \mathrm{~mL}$ glass vials at $4{ }^{\circ} \mathrm{C}$ in refrigerator. The size, PDI and zetapotential of the stored formulations were assessed at predefined time intervals (fresh samples, 1, 2, 3 and 4 weeks after production).

\subsubsection{Dissolution studies}

The dissolution studies were carried out in a glass vessel containing magnetic stirrer. HBSS with HEPES (Hanks' balanced salt solution with N-2-hydroxyethylpiperazine-N'-2ethanesulfonic acid) buffer $\mathrm{pH} 7.4$ solution with $4 \%$ Tween 80 was used as dissolution medium. For the dissolution study, the aqueous dispersions of the formulations $(2 \mathrm{~mL})$ were put into the dissolution vessel containing dissolution medium. The study was conducted at $37^{\circ} \mathrm{C}$, the amount of the medium was $250 \mathrm{~mL}$, and stirring speed was $100 \mathrm{rpm}$. The aliquots were sampled at predefined time intervals $(0,15 \mathrm{~min}, 30 \mathrm{~min}, 45 \mathrm{~min}, 60 \mathrm{~min}, 1.5 \mathrm{~h}, 2 \mathrm{~h}, 3$ 
h, 4 h, 6 h, 8 h, 12 h, and 24 h), and replaced with the same volume of fresh buffer. Samples withdrawn from the dissolution media were analyzed as such using a spectrophotometer with a wavelength of $480 \mathrm{~nm}$ for DOX, and HPLC for PXT.

Dissolution studies for the pure drugs in powdered form were carried out using the same protocol.

\subsubsection{High performance liquid chromatography (HPLC)}

In the HPLC method, the column used for the PXT detection was C18 $(4.6 \times 150 \mathrm{~mm} \times 5$ mm, Supelco Discovery C18, Phenomenex, USA), and the mobile phase used consist of water and acetonitrile $(53: 47, \mathrm{v} / \mathrm{v})$. The flow rate was $1.0 \mathrm{~mL} / \mathrm{min}$, the temperature of column was $25^{\circ} \mathrm{C}$, and the wavelength used for the drug detection was $227 \mathrm{~nm}$. The injected volume of the drug solution was $20 \mu \mathrm{L}$.

\subsubsection{Cell culturing}

The MCF-7 breast cancer cells and PC3-MM2 human prostate cancer cells were grown in $75 \mathrm{~cm}^{2}$ culture flasks (Corning Inc. Life Sciences, USA). The incubation was performed in $5 \% \mathrm{CO}_{2}$ in a gas incubator (Heraeus Instruments $\mathrm{GmbH}$, Germany) at $37^{\circ} \mathrm{C}$ with $95 \%$ relative humidity. MCF-7 and PC3-MM2 cells were cultured in DMEM culture medium. The medium was supplemented with $1 \%$ non-essential amino acids (NEAA), $10 \%(\mathrm{v} / \mathrm{v})$ fetal bovine serum (FBS), 1\% L-glutamine, and 1\% penicillin/streptomycin (PEST). Cells were thawed from the frozen stock and sub cultured at $80 \%$ confluency.

\subsubsection{Cytotoxicity studies}

The in vitro cytotoxicity of the niosomes was studied for the evaluation of the safety of the formulations. The viability of MCF-7 and PC3-MM2 cells was determined using an ATPbased cell viability kit. 
Briefly, the cells were seeded in 96-well plates at a concentration of $2 \times 10^{5}$ cells $/ \mathrm{mL}(100$ $\mu \mathrm{L})$ and incubated overnight for the attachment. The niosomes were prepared in the medium with the concentration ranging from 25 to $1000 \mu \mathrm{g} / \mathrm{mL}$. The cell medium in 96well plates was then replaced with the $100 \mu \mathrm{L}$ of the fresh medium containing niosomes and incubated for $24 \mathrm{~h}$. After the incubation, the ATP-based viability was measured by further adding $100 \mu \mathrm{L}$ of reagent assay into each well (CellTiter-Glo Luminescent Cell Viability Assay, Promega, USA). The luminescence was measured with a Varioskan Flash plate reader (Thermo Fisher Scientific Inc., USA). The cells incubated with the cell culture medium and with Triton X-100 (1\%) were measured as positive and negative controls, respectively. All the measurements were made triplicate.

\subsubsection{Cell uptake studies}

For qualitative evaluation of the cellular uptake of niosomes, $200 \mu \mathrm{L}$ of MCF-7 and PC3MM2 cells were seeded into an 8-chamber slides (Nunc Lab-Tek II Chamber Slide System, Thermo scientific, Inc., USA) at a density of $2.5 \times 10^{4}$ cells per well, and incubated at $37^{\circ} \mathrm{C}$ overnight for proper attachment of the cells to the chamber. After the removal of the cell media, the cells were washed with HBSS-HEPES buffer (pH 7.4). Fluorescein isothiocyanate (FITC) labeled niosomes were prepared by loading the FITC during the aforementioned method for the preparation of drug loaded niosomes. $200 \mu \mathrm{L}$ of FITC labelled niosome suspension with different concentrations were added in each chamber and incubated at $37^{\circ} \mathrm{C}$ for $6 \mathrm{~h}$ time. After incubation, the cells were washed thrice with HBSSHEPES buffer ( $\mathrm{pH} 7.4)$ in order to remove the free niosomes and the cell membrane was stained with CellMask Deep Red ( $5 \mu \mathrm{L} / \mathrm{mL}$, Invitrogen, USA) for 3 min at $37^{\circ} \mathrm{C}$. Cells were again washed with HBSS-HEPES buffer ( $\mathrm{pH} 7.4$ ), washed and fixed with $4 \%$ paraformaldehyde (PFA) for $15 \mathrm{~min}$, and washed with HBSS-HEPES buffer (pH 7.4). After the nuclei was stained by adding $200 \mu \mathrm{L}$ of DAPI (4',6-diamidino-2-phenylindole, 2.8 
$\mu \mathrm{L} / \mathrm{mL}$, Thermo Scientific, USA) for $5 \mathrm{~min}$, cells were washed again and stored with 200 $\mu \mathrm{L}$ of HBSS-HEPES buffer (pH 7.4). The interaction of the niosomes with the cells was studied by a Leica SP5 inverted confocal microscope (Leica Microsystems, Germany), using a $63 \times 1.2-0.6$ oil immersion objective.

\subsubsection{Anti-proliferation assay}

The in vitro cell growth inhibition of the drug-loaded niosomes was evaluated against the MCF-7 and PC3-MM2 cancer cells by cell proliferation experiments. The anti-proliferation effects of free DOX, PXT, and drugs loaded niosomal formulations, containing different concentrations of drugs $(25-500 \mu \mathrm{g} / \mathrm{mL})$ were measured using the previously described protocol for the cytotoxicity studies. All the experiments were repeated three times.

\subsubsection{Statistical analysis}

The statistical significance was determined using Student's t test (two-tailed); $\mathrm{P}<0.05$ was considered statistically significant in all the analyses (95\% confidence level).

\section{Results and Discussion}

\subsection{Preparation of niosomes}

Niosomes were prepared with constant amounts of Span 60, cholesterol and drug materials (DOX and/or PXT), but with varying amounts of Pluronic L121 and DCP. In the niosome structure, DOX as hydrophilic drug is encapsulated inside the vesicles and PXT as hydrophobic drug is entrapped into the bilayer structure. Cholesterol is situated in the bilayer structure rigidifying it and minimizing drug leakage, and making the drug release controlled. Three different levels of the amount of Pluronic L121 and DCP were studied. 
The central point composition for factorial design was selected to be $290 \mathrm{mg}$ of Pluronic L121 and $1 \mathrm{mg}$ of DCP. The exact compositions of all the niosome formulations are shown in Table 1.

3.2 Size and surface properties of niosomes

In this study, physicochemical parameters, size $(<350 \mathrm{~nm})$, PDI $(<0.5)$ and zeta-potential $(<-30 \mathrm{mV})$ were considered as critical quality attributes, CQAs, for the niosomes. The PDI value below 0.5 indicates low level of aggregation. Similarly, the zeta-potential value smaller than $-30 \mathrm{mV}$ means more stable niosomes with less aggregation tendency due to the electric repulsive forces (Mahale et al., 2012). DCP was added to the composition in order to reach lower zeta-potential values. Previous studies have shown that niosomal formulations containing cholesterol are rigid, stable and intact, while niosomes without cholesterol forms gel like systems, and for that reason cholesterol was added to all the compositions (Somjid et al., 2018).

The non-ionic amphiphiles having hydrophilic-lipophilic balance (HLB) value greater than 11, hinder the vesicular formation. Span 60 , selected for this study, forms rigid, stable and large in size niosomes having high drug entrapment efficiencies due to larger particle size (Basiri et al., 2017). Finally, the non-ionic polymeric surfactant, Pluronic L121, has the capability to entrap hydrophobic drugs, like PXT, efficiently inside the niosomes. And, it forms monodisperse and stable niosomes (Lee et al., 2005; Lee et al., 2011; Abdelbary and Tadros, 2013).

The negative charge-imparting agent in this study was DCP. It gives for particle population low polydispersity values, due to strong inter-niosomal repulsive forces induced by the high surface charge (Helal et al., 2015). In this study, the size of all the niosomal formulations (with and without drug) were between $137 \mathrm{~nm}$ and $893 \mathrm{~nm}$. The PDI ranged between 0.383 
and 0.725 and zeta-potential values were from $-26.5 \mathrm{mV}$ to $-49.1 \mathrm{mV}$ (Table 2). Based on the size, size deviation and surface charge measurements, most of the studied niosomal formulations filled the above mentioned CQA requirements. 
Table 2. Particle sizes, PDI values, zeta-potentials, and drug entrapment efficiency values for studied niosomal formulations.

\begin{tabular}{cccccc}
\hline Formulation & Size $(\mathbf{n m})$ & PDI & $\begin{array}{c}\text { Zeta-potential } \\
(\mathbf{m V})\end{array}$ & \%EE $(\mathbf{D O X})$ & \%EE $(\mathbf{P X T})$ \\
\hline D1 & $204.4 \pm 8.7$ & $0.470 \pm 0.069$ & $-34.9 \pm 0.5$ & $92.90 \pm 0.13$ & - \\
D2 & $182.9 \pm 20.7$ & $0.423 \pm 0.009$ & $-36.8 \pm 1.6$ & $93.49 \pm 0.20$ & - \\
D3 & $241.1 \pm 37.0$ & $0.501 \pm 0.007$ & $-34.3 \pm 2.6$ & $92.32 \pm 0.18$ & - \\
D4 & $202.1 \pm 18.0$ & $0.510 \pm 0.033$ & $-46.9 \pm 1.1$ & $92.99 \pm 0.20$ & - \\
D5 & $190.1 \pm 7.2$ & $0.491 \pm 0.048$ & $-39.6 \pm 1.1$ & $92.73 \pm 0.20$ & - \\
P1 & $195.3 \pm 21.5$ & $0.462 \pm 0.055$ & $-35.9 \pm 2.0$ & - & $99.96 \pm 0.00$ \\
P2 & $178.1 \pm 3.0$ & $0.417 \pm 0.027$ & $-43.8 \pm 0.6$ & - & $99.98 \pm 0.00$ \\
P3 & $169.3 \pm 19.2$ & $0.435 \pm 0.098$ & $-49.1 \pm 1.3$ & - & $99.98 \pm 0.00$ \\
P4 & $180.6 \pm 7.2$ & $0.447 \pm 0.076$ & $-38.9 \pm 3.2$ & - & $99.96 \pm 0.00$ \\
P5 & $173.5 \pm 42.6$ & $0.383 \pm 0.025$ & $-47.0 \pm 1.3$ & - & $99.97 \pm 0.00$ \\
DP1 & $176.7 \pm 11.4$ & $0.493 \pm 0.010$ & $-27.7 \pm 2.0$ & $91.24 \pm 0.33$ & $99.99 \pm 0.00$ \\
DP2 & $156.6 \pm 10.2$ & $0.450 \pm 0.009$ & $-26.5 \pm 0.7$ & $92.58 \pm 0.39$ & $99.99 \pm 0.00$ \\
DP3 & $147.6 \pm 14.7$ & $0.448 \pm 0.095$ & $-32.1 \pm 0.8$ & $92.31 \pm 0.21$ & $99.99 \pm 0.00$ \\
DP4 & $168.2 \pm 11.8$ & $0.481 \pm 0.072$ & $-39.0 \pm 2.4$ & $92.39 \pm 0.13$ & $99.99 \pm 0.00$ \\
DP5 & $137.1 \pm 4.1$ & $0.437 \pm 0.046$ & $-41.8 \pm 2.6$ & $93.67 \pm 0.27$ & $99.99 \pm 0.00$ \\
E1 & $195.6 \pm 12.8$ & $0.492 \pm 0.047$ & $-27.5 \pm 0.9$ & - & - \\
E2 & $236.3 \pm 36.0$ & $0.391 \pm 0.105$ & $-27.5 \pm 0.9$ & - & - \\
E3 & $443.5 \pm 86.7$ & $0.469 \pm 0.037$ & $-34.9 \pm 3.4$ & - & - \\
E4 & $300.5 \pm 36.6$ & $0.448 \pm 0.034$ & $-38.8 \pm 0.3$ & - & - \\
E5 & $893.6 \pm 135.5$ & $0.725 \pm 0.117$ & $-39.9 \pm 5.2$ & - & - \\
\hline
\end{tabular}

Three batches failed to fulfill the PDI criteria, but two of these (D3 and D4) were very close to the critical value (0.501 and 0.510). D3 formulation did not contain DCP, and D4 formulation contained the lowest amount of Pluronic L121 (246 mg). All the drug loaded niosomes were small in size, below $250 \mathrm{~nm}$, but the size criteria was not fulfilled with two batches of empty niosomes E3 and E5 (E3 composition without DCP and E5 with the highest amount of Pluronic L121 (334 mg)). The zeta-potential value was not negative enough with four batches. However, the zeta-potential of these four batches was from $26.5 \mathrm{mV}$ to $-27.7 \mathrm{mV}$, which can still be considered high enough value to stabilize the niosomes. This was also confirmed in the stability studies, where all the batches showed good stability.

\subsection{Drug entrapment efficiency (\%EE)}

The drug entrapment efficiency is very important property for drug formulations. In this study, the formulations that contained DOX, the \%EE ranged between $92.32 \%$ and $93.49 \%$. 
The formulations containing only PXT, the \%EE was even higher, being between $99.96 \%$ to $99.98 \%$. With the formulations containing both DOX and PXT, entrapment efficiencies for DOX varied between $91.24 \%$ and $93.67 \%$, while PXT had in all the formulations 99.99\% entrapment efficiency value. Accordingly, the entrapment efficiencies were in very high level, especially entrapment of PXT was very close to $100 \%$, and no real differences were seen in the entrapment efficiency values between different compositions with PXT.

The high entrapment efficiency of PXT was due to its very low water solubility. The affinity of the drug inside the niosomes is very high due to its lipophilicity: it has very high tendency to escape from the outer aqueous phase to niosomes. Aqueous solubility of DOX is higher and it can partly be left into the aqueous phase, shown by the slightly lower entrapment efficiency values. However, the drug entrapment efficiency values were generally high for both the drugs in all the tested compositions, and it can be concluded that the compositions of niosome formulations have very minor effect on the entrapment efficiencies.

In all the studied drug loaded formulations, the mean size of the niosomes was from 137 $\mathrm{nm}$ to $241 \mathrm{~nm}$. The composition is affecting the particle size of the niosomes, and particle size can also affect the entrapment efficiency: it is typical that larger particles are able to entrap the drugs more efficiently. However, in this study the changes in particle sizes were that small that any conclusions of its impact on the drug entrapment could not be withdrawn.

Some examples of TEM images for niosomal formulations are shown in Figure 1. Based on the TEM analysis, the niosomes are close to spherical in shape and the sizes are corresponding well with the ones measured by DLS. No pure drug crystals were seen in TEM figures which is in accordance with the high drug entrapment values. This is also 
supported by the fact that the PDI values of the empty niosomes were in the same level or even higher than corresponding values for drug loaded niosomes.

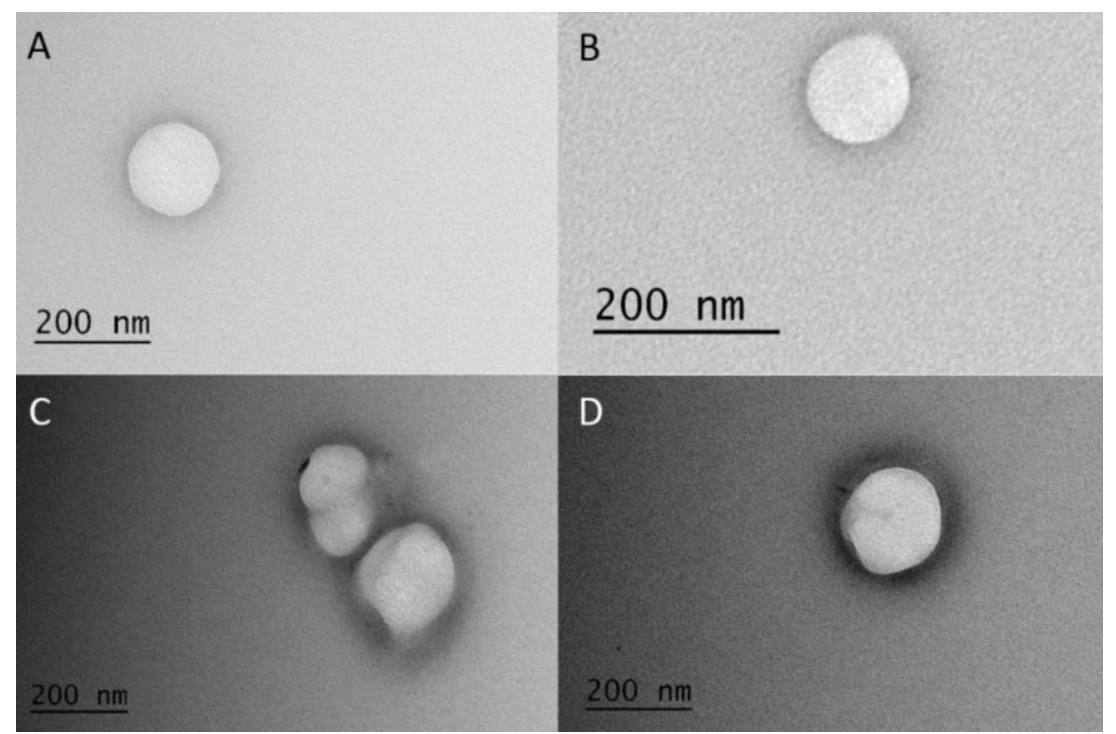

Figure 1. TEM images of E1 (figures A and B), and E2 (figures C and D) compositions of niosome formulations.

\subsection{Stability studies}

For stability testing purposes, all the niosomes were stored at $4^{\circ} \mathrm{C}$ for 4 weeks, and all the formulations were studied taking into account the above-mentioned CQAs as critical stability parameters. The niosomes (without drug) tend to increase in the size, but after one week in storage, they became stable. It is typical that after the process stress (here sonication), particle size can change a little bit before reaching a constant value. This is due to the relaxation process after the stress (sonication) phase is over. The particle sizes of the empty niosomes ranged between $195 \mathrm{~nm}$ and $893 \mathrm{~nm}$ with PDI values from 0.39 to 0.72 , and zeta-potentials ranging from $-27 \mathrm{mV}$ to $-39 \mathrm{mV}$ as shown in Table 3. During the whole 
storage time (4 weeks), all the drug-loaded niosomes were stable and only a little fluctuation in size was seen. Most stable formulations were the center point formulations D1, P1 and DP1 as well as formulations containing $2 \mathrm{mg}$ of DCP, e.g. D2, P2 and DP2. The zeta-potential of the formulations D1, D2, P1, P2, DP1 and DP2 were deviating from the predetermined CQAs and was close to the limiting values. However, the low PDI values and stable particle sizes suggested a low level of aggregation and high stability of the niosomes, demonstrating good quality of these batches, too. The niosomes remained stable with low PDI values during the stability testing time as indicated in Table 3. The results indicated that higher quantities of DCP ( 1 or $2 \mathrm{mg}$ ) and medium quantity of Pluronic L121 (290 mg) showed the best stability of niosomes. 
Table 3. Particle size, PDI and zeta-potential values of fresh (just after the production) and stored (at $4^{\circ} \mathrm{C}$ ) niosomal formulations.

\begin{tabular}{|c|c|c|c|c|c|c|c|c|c|c|c|}
\hline Time & Parameters & E1 & E2 & $\mathbf{E 3}$ & E4 & E5 & D1 & D2 & D3 & D4 & D5 \\
\hline \multirow{3}{*}{ 昰高 } & Size (nm) & $195.6 \pm 12.8$ & $236.3 \pm 36.0$ & $443.5 \pm 86.7$ & $300.5 \pm 36.6$ & $893.6 \pm 135.5$ & $204.4 \pm 8.7$ & $182.9 \pm 20.7$ & $241.1 \pm 37.0$ & $202.1 \pm 18.0$ & $190.1 \pm 7.2$ \\
\hline & PDI & $0.492 \pm 0.047$ & $0.391 \pm 0.105$ & $0.469 \pm 0.037$ & $0.448 \pm 0.034$ & $0.725 \pm 0.117$ & $0.470 \pm 0.069$ & $0.423 \pm 0.009$ & $0.501 \pm 0.007$ & $0.510 \pm 0.033$ & $0.491 \pm 0.048$ \\
\hline & Zeta-potential (mV) & $-27.5 \pm 0.9$ & $-27.5 \pm 0.9$ & $-34.9 \pm 3.4$ & $-38.8 \pm 0.3$ & $-39.9 \pm 5.2$ & $-34.9 \pm 0.5$ & $-36.8 \pm 1.6$ & $-34.3 \pm 2.6$ & $-46.9 . \pm 1.1$ & $-39.6 \pm 1.1$ \\
\hline \multirow{3}{*}{ 总 } & Size (nm) & $223.4 \pm 11.4$ & $175.8 \pm 5.8$ & $294.4 \pm 8.0$ & $199.3 \pm 5.4$ & $244.8 \pm 7.6$ & $200.0 \pm 7.0$ & $185.2 \pm 10.1$ & $240.1 \pm 36.2$ & $200.2 \pm 10.2$ & $192.1 \pm 9.1$ \\
\hline & PDI & $0.340 \pm 0.046$ & $0.198 \pm 0.040$ & $0.362 \pm 0.038$ & $0.282 \pm 0.014$ & $0.535 \pm 0.020$ & $0.341 \pm 0.011$ & $0.314 \pm 0.071$ & $0.430 \pm 0.017$ & $0.490 \pm 0.033$ & $0.451 \pm 0.011$ \\
\hline & Zeta-potential (mV) & $-23.8 \pm 0.9$ & $-26.9 \pm 0.9$ & $-28.2 \pm 1.0$ & $-28.5 \pm 0.6$ & $-30.4 \pm 0.5$ & $-32.3 \pm 0.1$ & $-34.0 \pm 1.5$ & $-34.1 \pm 2.2$ & $-46.1 . \pm 2.2$ & $-36.8 \pm 2.1$ \\
\hline \multirow{3}{*}{ 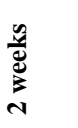 } & Size $(\mathbf{n m})$ & $191.9 \pm 3.3$ & $178.7 \pm 3.6$ & $347.4 \pm 9.0$ & $212.7 \pm 6.1$ & $245.2 \pm 5.5$ & $201.0 \pm 4.0$ & $183.5 \pm 9.1$ & $241.3 \pm 20.2$ & $207.6 \pm 12.2$ & $193.1 \pm 5.2$ \\
\hline & PDI & $0.168 \pm 0.039$ & $0.169 \pm 0.028$ & $0.375 \pm 0.030$ & $0.299 \pm 0.022$ & $0.543 \pm 0.015$ & $0.340 \pm 0.019$ & $0.310 \pm 0.011$ & $0.415 \pm 0.011$ & $0.450 \pm 0.011$ & $0.410 \pm 0.034$ \\
\hline & Zeta-potential (mV) & $-23.8 \pm 0.9$ & $-28.1 \pm 2.2$ & $-29.5 \pm 2.5$ & $-28.2 \pm 3.0$ & $-26.3 \pm 0.5$ & $-32.5 \pm 0.1$ & $-33.9 \pm 1.6$ & $-33.5 \pm 3.3$ & $-45.0 \pm 3.1$ & $-36.2 \pm 3.1$ \\
\hline \multirow{3}{*}{ 告 } & Size (nm) & $199.4 \pm 4.9$ & $172.2 \pm 1.7$ & $296.3 \pm 12.6$ & $208.7 \pm 4.6$ & $291.6 \pm 8.3$ & $203.1 \pm 2.6$ & $182.9 \pm 11.2$ & $253.6 \pm 26.4$ & $215.2 \pm 12.2$ & $201.3 \pm 5.1$ \\
\hline & PDI & $0.213 \pm 0.011$ & $0.151 \pm 0.033$ & $0.320 \pm 0.051$ & $0.324 \pm 0.020$ & $0.532 \pm 0.051$ & $0.331 \pm 0.054$ & $0.315 \pm 0.039$ & $0.420 \pm 0.027$ & $0.450 \pm 0.011$ & $0.421 \pm 0.091$ \\
\hline & Zeta-potential (mV) & $-27.2 \pm 0.4$ & $-28.9 \pm 0.5$ & $-28.6 \pm 1.5$ & $-31.6 \pm 2.2$ & $-28.9 \pm 0.2$ & $-32.7 \pm 0.1$ & $-33.5 \pm 3.2$ & $-34.0 \pm 3.3$ & $-45.1 . \pm 2.1$ & $-36.2 \pm 7.1$ \\
\hline \multirow{3}{*}{ 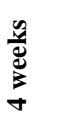 } & Size (nm) & $186.8 \pm 1.6$ & $182.0 \pm 2.7$ & $311.8 \pm 8.1$ & $190.0 \pm 2.5$ & $255.8 \pm 34.8$ & $201.2 \pm 4.0$ & $183.5 \pm 9.1$ & $260.6 \pm 26.4$ & $221.2 \pm 12.2$ & $210.3 \pm 5.1$ \\
\hline & PDI & $0.138 \pm 0.025$ & $0.226 \pm 0.016$ & $0.364 \pm 0.042$ & $0.208 \pm 0.037$ & $0.534 \pm 0.078$ & $0.340 \pm 0.019$ & $0.310 \pm 0.011$ & $0.420 \pm 0.027$ & $0.450 \pm 0.011$ & $0.421 \pm 0.091$ \\
\hline & Zeta-potential (mV) & $-25.5 \pm 1.3$ & $-27.3 \pm 1.5$ & $-26.7 \pm 0.2$ & $-27.8 \pm 1.0$ & $-29.8 \pm 1.1$ & $-32.5 \pm 0.1$ & $-33.2 \pm 1.7$ & $-34.0 \pm 3.3$ & $-45.1 \pm 2.1$ & $-36.2 \pm 7.1$ \\
\hline \multirow{4}{*}{ 预 } & & P1 & $\mathbf{P 2}$ & P3 & $\mathbf{P 4}$ & P5 & DP1 & DP2 & DP3 & DP4 & DP5 \\
\hline & Size (nm) & $195.3 \pm 21.5$ & $178.1 \pm 3.0$ & $169.3 \pm 19.2$ & $180.6 \pm 7.2$ & $173.5 \pm 42.6$ & $176.7 \pm 11.4$ & $156.6 \pm 10.2$ & $147.6 \pm 14.7$ & $168.2 \pm 12.0$ & $137.1 \pm 4.1$ \\
\hline & PDI & $0.462 \pm 0.055$ & $0.417 \pm 0.027$ & $0.435 \pm 0.098$ & $0.447 \pm 0.076$ & $0.383 \pm 0.025$ & $0.493 \pm 0.010$ & $0.450 \pm 0.009$ & $0.448 \pm 0.095$ & $0.481 \pm 0.072$ & $0.437 \pm 0.046$ \\
\hline & Zeta-potential (mV) & $-35.9 \pm 2.0$ & $-43.8 \pm 0.6$ & $-49.1 \pm 1.3$ & $-38.9 \pm 3.2$ & $-47.0 \pm 1.4$ & $-27.7 \pm 2.0$ & $-26.5 \pm 0.8$ & $-32.1 \pm 1.0$ & $-39.0 \pm 2.4$ & $-41.8 \pm 2.7$ \\
\hline \multirow{3}{*}{ हू } & Size (nm) & $193.2 \pm 11.1$ & $175.2 \pm 5.6$ & $165.3 \pm 12.1$ & $181.5 \pm 5.2$ & $170.5 \pm 22.7$ & $171.7 \pm 10.1$ & $157.2 \pm 10.3$ & $146.3 \pm 11.6$ & $167.5 \pm 12.8$ & $141.1 \pm 4.1$ \\
\hline & PDI & $0.342 \pm 0.029$ & $0.317 \pm 0.011$ & $0.397 \pm 0.018$ & $0.417 \pm 0.016$ & $0.313 \pm 0.011$ & $0.393 \pm 0.032$ & $0.390 \pm 0.019$ & $0.380 \pm 0.095$ & $0.430 \pm 0.011$ & $0.430 \pm 0.146$ \\
\hline & Zeta-potential (mV) & $-32.2 \pm 3.0$ & $-39.1 \pm 0.6$ & $-45.1 \pm 7.3$ & $-35.3 \pm 1.2$ & $-45.0 \pm 1.3$ & $-27.5 \pm 1.4$ & $-25.5 \pm 0.4$ & $-31.2 \pm 0.6$ & $-35.1 \pm 2.1$ & $-39.2 \pm 5.7$ \\
\hline \multirow{3}{*}{ 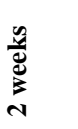 } & Size (nm) & $195.0 \pm 10.2$ & $172.3 \pm 5.2$ & $170.3 \pm 22.2$ & $186.5 \pm 10.1$ & $175.9 \pm 12.2$ & $170.7 \pm 10.2$ & $155.0 \pm 10.4$ & $149.4 \pm 15.2$ & $168.9 \pm 21.1$ & $144.6 \pm 7.2$ \\
\hline & PDI & $0.331 \pm 0.039$ & $0.310 \pm 0.011$ & $0.410 \pm 1.018$ & $0.419 \pm 0.026$ & $0.321 \pm 0.021$ & $0.389 \pm 0.011$ & $0.379 \pm 0.013$ & $0.389 \pm 0.011$ & $0.410 \pm 0.025$ & $0.429 \pm 0.210$ \\
\hline & Zeta-potential (mV) & $-32.0 \pm 3.7$ & $-39.28 \pm 0.2$ & $-45.1 \pm 4.3$ & $-35.6 \pm 2.1$ & $-43.6 \pm 1.5$ & $-27.2 \pm 1.1$ & $-26.1 \pm 2.0$ & $-31.2 \pm 0.1$ & $-35.9 \pm 2.2$ & $-39.5 \pm 3.5$ \\
\hline \multirow{3}{*}{ m } & Size (nm) & $191.1 \pm 10.2$ & $175.9 \pm 5.1$ & $198.3 \pm 22.2$ & $195.5 \pm 8.2$ & $189.9 \pm 10.2$ & $172.3 \pm 15.1$ & $154.3 \pm 10.2$ & $170.2 \pm 10.4$ & $192.3 \pm 19.3$ & $177.2 \pm 7.1$ \\
\hline & PDI & $0.310 \pm 0.039$ & $0.335 \pm 0.011$ & $0.397 \pm 1.018$ & $0.410 \pm 0.026$ & $0.325 \pm 0.021$ & $0.390 \pm 0.012$ & $0.389 \pm 0.313$ & $0.391 \pm 0.321$ & $0.419 \pm 0.011$ & $0.437 \pm 0.217$ \\
\hline & Zeta-potential (mV) & $-32.9 \pm 4.0$ & $-39.3 \pm 0.5$ & $-45.6 \pm 4.3$ & $-35.2 \pm 2.3$ & $-44.9 \pm 1.4$ & $-27.9 \pm 3.1$ & $-26.5 \pm 3.4$ & $-31.4 \pm 1.2$ & $-35.5 \pm 2.3$ & $-39.3 \pm 4.2$ \\
\hline \multirow{3}{*}{ 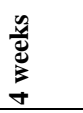 } & Size (nm) & $194.0 \pm 10.1$ & $176.4 \pm 5.3$ & $207.3 \pm 22.1$ & $210.5 \pm 8.1$ & $197.9 \pm 10.2$ & $171.7 \pm 15.1$ & $156.4 \pm 9.6$ & $183.4 \pm 15.1$ & $212.4 \pm 21.1$ & $191.6 \pm 7.3$ \\
\hline & PDI & $0.350 \pm 0.039$ & $0.310 \pm 0.011$ & $0.381 \pm 1.018$ & $0.410 \pm 0.026$ & $0.327 \pm 0.021$ & $0.370 \pm 0.012$ & $0.376 \pm 0.213$ & $0.389 \pm 0.011$ & $0.431 \pm 0.025$ & $0.423 \pm 0.210$ \\
\hline & Zeta-potential (mV) & $-32.3 \pm 4.2$ & $-39.3 \pm 0.6$ & $-45.9 \pm 4.2$ & $-35.2 \pm 2.2$ & $-43.5 \pm 1.4$ & $-27.4 \pm 3.1$ & $-26.9 \pm 2.1$ & $-31.2 \pm 0.2$ & $-35.2 \pm 2.3$ & $-39.7 \pm 3.6$ \\
\hline
\end{tabular}




\subsection{Interaction studies}

\subsubsection{ATR-FTIR spectroscopy}

The ATR-FTIR spectroscopy is a pre-formulation study for the evaluation of compatibility between the formulation ingredients. The ATR-FTIR spectra of DOX, PXT, and all the other ingredients individually, the physical mixtures of formulations, and their corresponding niosomal formulations are shown in Figure 2.

SP1 nesomes

Figure 2. ATR-FTIR spectra of different raw materials, physical mixtures and niosomal formulations.

The pure DOX showed peaks at $3456 \mathrm{~cm}^{-1}$ and $3335 \mathrm{~cm}^{-1}$ due to the N-H stretching of primary amine and $\mathrm{O}-\mathrm{H}$ stretching, respectively. The peaks at $868 \mathrm{~cm}^{-1}$ and $807 \mathrm{~cm}^{-1}$ were seen due to N-H group stretching (Majeed et al., 2013). The ATR-FTIR spectrum of PXT (pure drug) showed peaks between $3479 \mathrm{~cm}^{-1}$ to $3300 \mathrm{~cm}^{-1}$ due to the stretching of $\mathrm{N}-\mathrm{H}, \mathrm{CH}_{2}$ stretching peaks between $2976-2885 \mathrm{~cm}^{-1}, \mathrm{C}=\mathrm{O}$ stretching at $1734 \mathrm{~cm}^{-1}$, the amide bond stretching at $1647 \mathrm{~cm}^{-1}$, ester bond and C-N stretching at $1254 \mathrm{~cm}^{-1}$ and $1276 \mathrm{~cm}^{-1}$, 
respectively, and peaks due to aromatic bonds were seen at 1647, 1074, 963 and $709 \mathrm{~cm}^{-1}$ (Martins et al., 2014). Span 60 gave peaks at $2916.75 \mathrm{~cm}^{-1}$ due to (-OH stretch, broad), $2849.58 \mathrm{~cm}^{-1}$ (-OH stretch, broad), a 5-membered cyclic ring peak at $1734.65 \mathrm{~cm}^{-1}$ and small peaks due to aliphatic groups from $1000-1200 \mathrm{~cm}^{-1}$ (Li et al., 2008) Pluronic L121 showed peak stretch at $2990 \mathrm{~cm}^{-1}$ of asymmetrical methyl $(\mathrm{C}-\mathrm{H})$, scissoring of C-H bondage at 1480 $\mathrm{cm}^{-1}$, symmetrical C-H bond at $1387 \mathrm{~cm}^{-1}$ and ether linkage (C-O-C) at $1120 \mathrm{~cm}^{-1}$ (Newman et al., 1998). Cholesterol showed ATR-FTIR peaks at $2931.41 \mathrm{~cm}^{-1}$ of acetyl group, 2866.83 $\mathrm{cm}^{-1}$ symmetric $-\mathrm{CH}_{3}$, at $1770.20 \mathrm{~cm}^{-1}$ and $1055.17 \mathrm{~cm}^{-1}$ due to vinyl group and R-O groups, respectively (Khan et al., 2015).

The ATR-FTIR of the physical mixtures of the optimized formulations and their relevant niosomal formulations were also studied. The spectra of both the physical mixtures and the niosomal formulations were similar and diffusion of the peaks were seen without peak shifting. Similar interactions between the ingredients was seen as in the earlier studies, where it was suggested that there was interactions between the Span 60 and cholesterol to give rigid and stable structure for niosomes (Nasseri, 2005). The found interaction is between the glycerol oxygen in Span molecule and $\beta-\mathrm{OH}$ group in cholesterol molecule; changes due to this interaction were seen in the spectrograms.

\subsubsection{Thermal analysis}

In the thermal analysis of Span 60, DCP, cholesterol, DOX and PXT, they showed endothermic characteristic melting peaks at $54^{\circ} \mathrm{C}, 78^{\circ} \mathrm{C}, 150^{\circ} \mathrm{C}, 205^{\circ} \mathrm{C}$ and $220^{\circ} \mathrm{C}$, respectively (Figure 3). The physical mixtures of formulations (D1, P1 and DP1) showed the slightly broader peaks at $38^{\circ} \mathrm{C}$ and $57^{\circ} \mathrm{C}$ which indicates the interaction between the Span 60 and cholesterol (Nasseri, 2005), as already mentioned related to the discussion of ATR-FTIR results, and which was also seen with the niosomal formulations D1, P1 and 
DP1. Also, a slightly broader peak was seen at $140{ }^{\circ} \mathrm{C}$ due to cholesterol. The peaks of membrane formers indicated mutual interactions, which resulted in more stable niosomes as indicated by the stability studies data and also by the entrapment efficiency values. The slightly broader peaks of drugs were seen in the range of $205^{\circ} \mathrm{C}$ to $230^{\circ} \mathrm{C}$ which indicated that the drugs were in their crystalline form as have been indicated in previous studies (Pawar and Vavia, 2016; Doustgani, 2017).

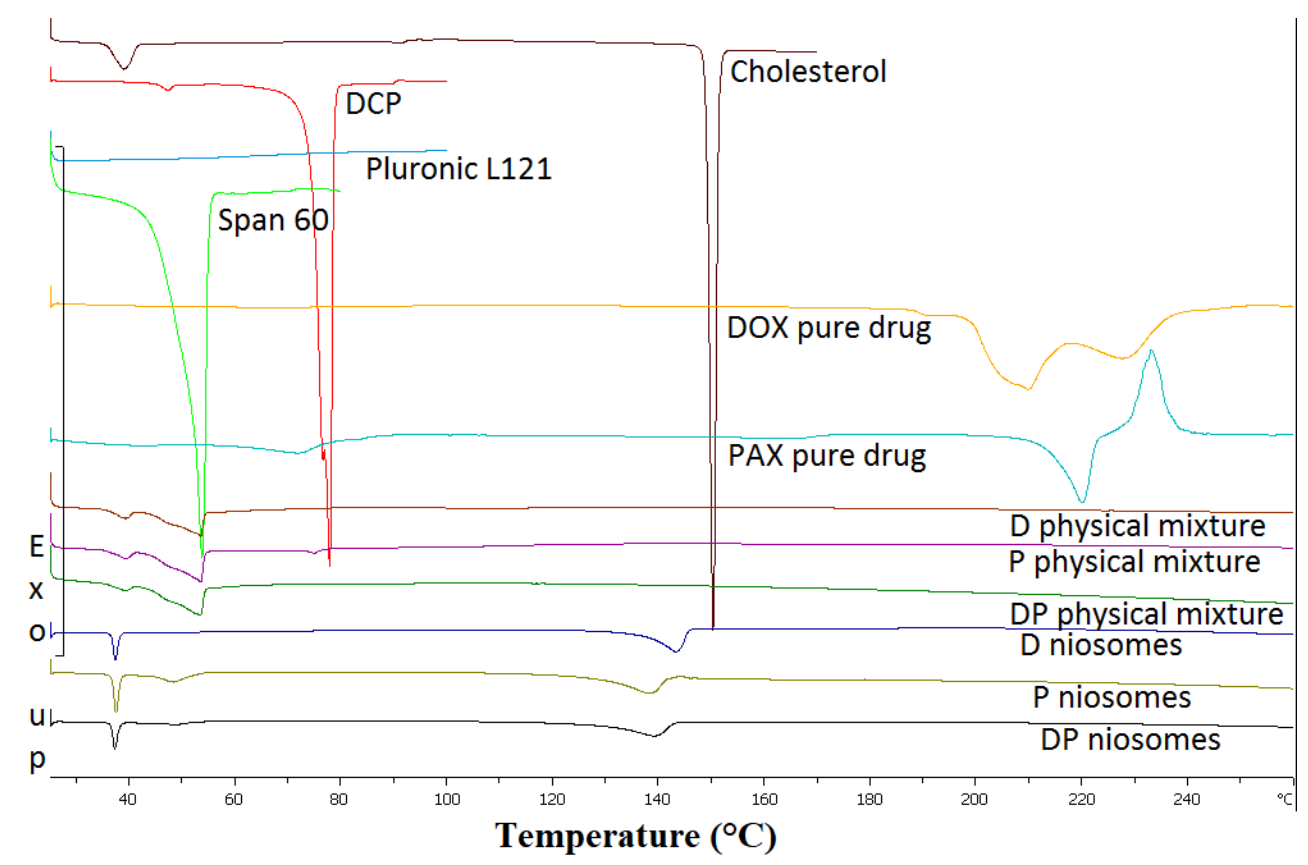

Figure 3. DSC thermograms of pure materials, physical mixtures and niosome formulations with PAX (P), DOX (D) and DOX and PAX (DP). 


\subsection{Drug Release Studies}

The in vitro drug release testing from the niosomes was performed in HBSS-HEPES (pH 7.4) with 4\% Tween 80 addition (Yang et al., 2007a; Figueiredo et al., 2017). The release profiles of DOX and PXT are summarized in Figure 4. From pure DOX powder, the dissolution was very fast. Drug release from the DOX niosomes was biphasic in nature: in the first phase, burst type of release was seen, which was followed by a constant sustained release phase. Dissolution rate from DOX niosomes was a little bit slower as compared to pure drug: from niosome formulations containing only DOX, drug release after $24 \mathrm{~h}$ time was approximately 93\%. Also, with the formulations containing both DOX and PXT, same kind of burst release was seen, but it was comparatively in lower level as compared to the formulations containing only DOX. Later, a constant sustained release of DOX from DOX and PXT loaded niosomes was observed reaching approximately $40 \%$ level within $24 \mathrm{~h}$.

Also with PXT, the biphasic release was observed. In the first phase, burst type release was seen and later a constant sustained release of PXT was seen from formulations containing only PXT reaching the approximated level of $26 \%$ within $24 \mathrm{~h}$. Again, with the formulations containing both DOX and PXT, a burst release of PXT was seen, but similarly to DOX release, it was less as compared to the formulations containing only PXT. In the second phase, a constant sustained release of PXT was observed reaching the approximated level of 18\% within $24 \mathrm{~h}$. All the PXT loaded niosomes showed much higher dissolution rates as compared to pure PXT.

The niosomal formulations containing both DOX and PXT showed lower drug release rates as compared with the formulations containing only one drug. 
A

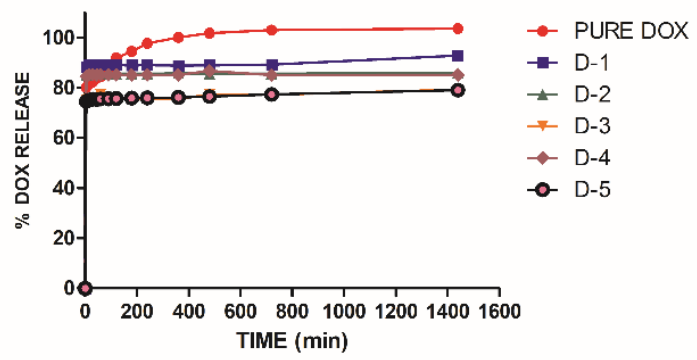

B

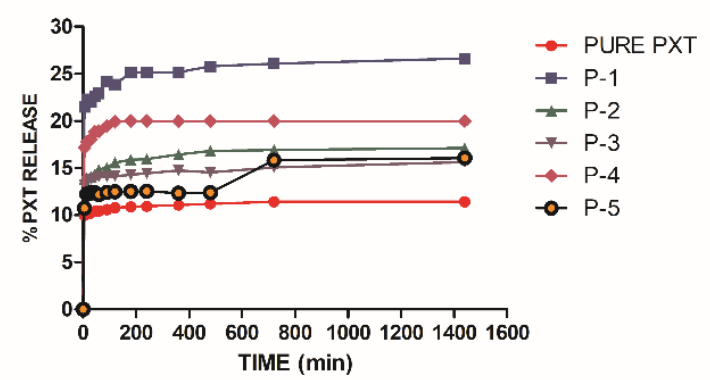

C

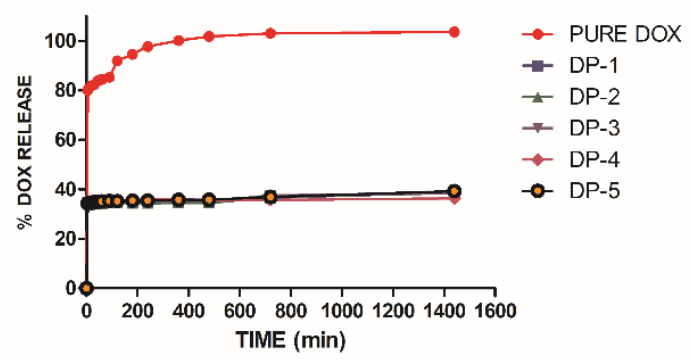

D

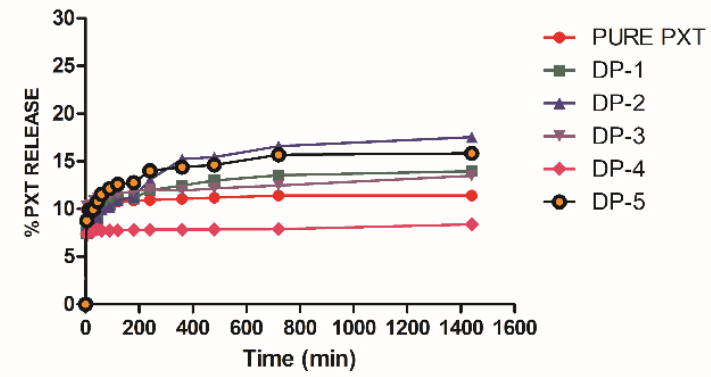

Figure 4. Drug release from formulations containing A) DOX, B) PXT, and formulations containing DOX and PXT, release of C) DOX, and D) PXT.

3.7 Cell viability assay

The biocompatibility of the niosomes is an important criterion for their application as a drug delivery system. In this study, the cytotoxicity of the niosomes was evaluated against MCF-7 and PC3-MM2 cell lines by incubating them for $24 \mathrm{~h}$ using different niosome concentrations as shown in Figure 5. With empty niosomes, weak concentration related effect on viability was noticed as compared to the negative control, but the viability of the cells with even the highest tested niosome concentration, $1000 \mu \mathrm{g} / \mathrm{ml}$ of niosomes, was not significantly lowered with either MCF-7 or PC3-MM2 cell lines (with both cell lines $\mathrm{p}>0.05)$. With all the studied concentrations, the viability values were higher with MCF-7 cells as compared to PC3-MM2 cells. 


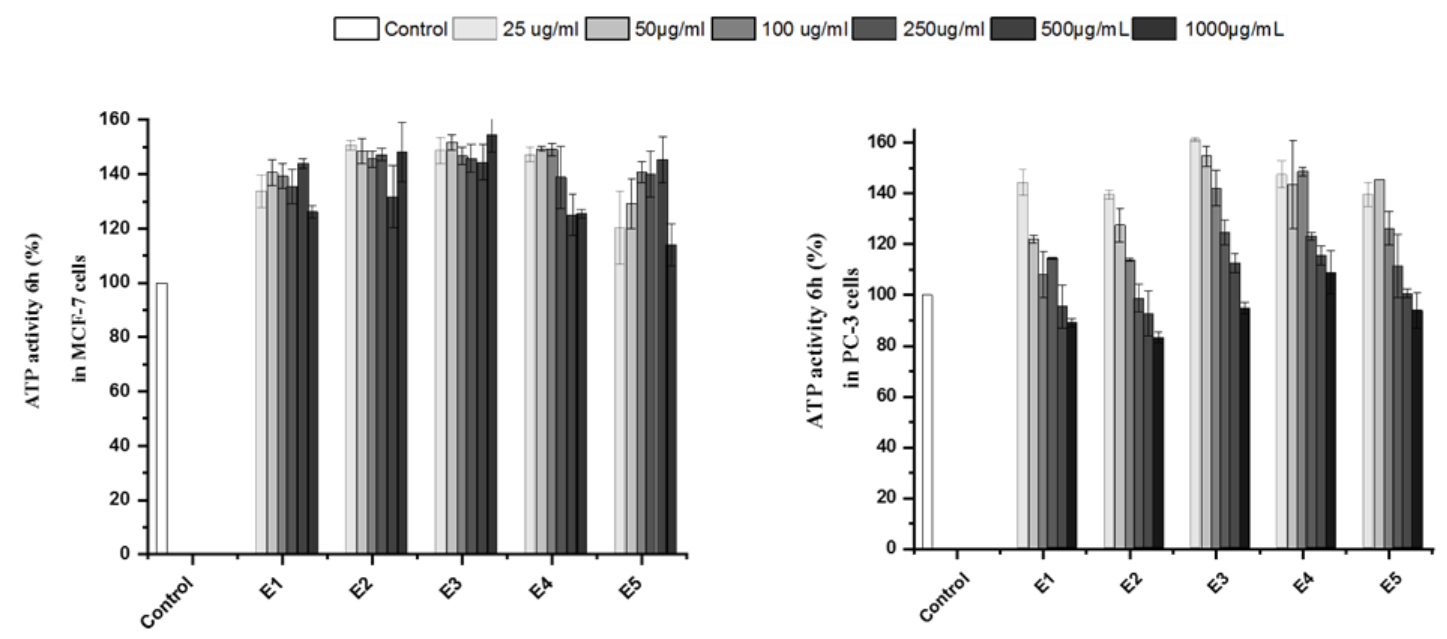

Figure 5. Cell viability assay of niosomal formulations against MCF-7 and PC3-MM2 cells after $24 \mathrm{~h}$ incubation.

\subsection{Cell uptake studies}

The cell uptake efficiency of the anticancer drug loaded niosomes is an important factor for the estimation of potency of drug formulations. A fluorescent compound, FITC, was incorporated into the niosomes, cell membranes were stained with CellMask Deep Red and nuclei with DAPI. The confocal images (Figure 6) of MCF-7 and PC3-MM2 cell lines were examined by incubating them at $37^{\circ} \mathrm{C}$ for $6 \mathrm{~h}$. Based on the confocal images, the niosomes were taken up by the cells and they were accumulated into the cytoplasm of the cells successfully. In earlier studies, it has been shown that the internalization of niosomes can be facilitated by the presence of lipid content of niosomes and its interaction with the lipid membrane of the cells (Guo et al., 2015; Shi et al., 2015; Tahir et al., 2017). The size of the niosomes also affects internalization and drug delivery into the cells. In the present study, the smallest size fractions $(<200 \mathrm{~nm})$ of niosomes had better penetration due to having small size, and the larger niosomes had less penetration capacity, which has also been described in previous studies (Shahbazi et al., 2014; Khutale and Casey, 2017). The formulation E2 showed the best internalization of the niosomes when compared with other 
formulations. In other formulations, part of the niosomes remained outside of the cells and adhered to cell surfaces. 

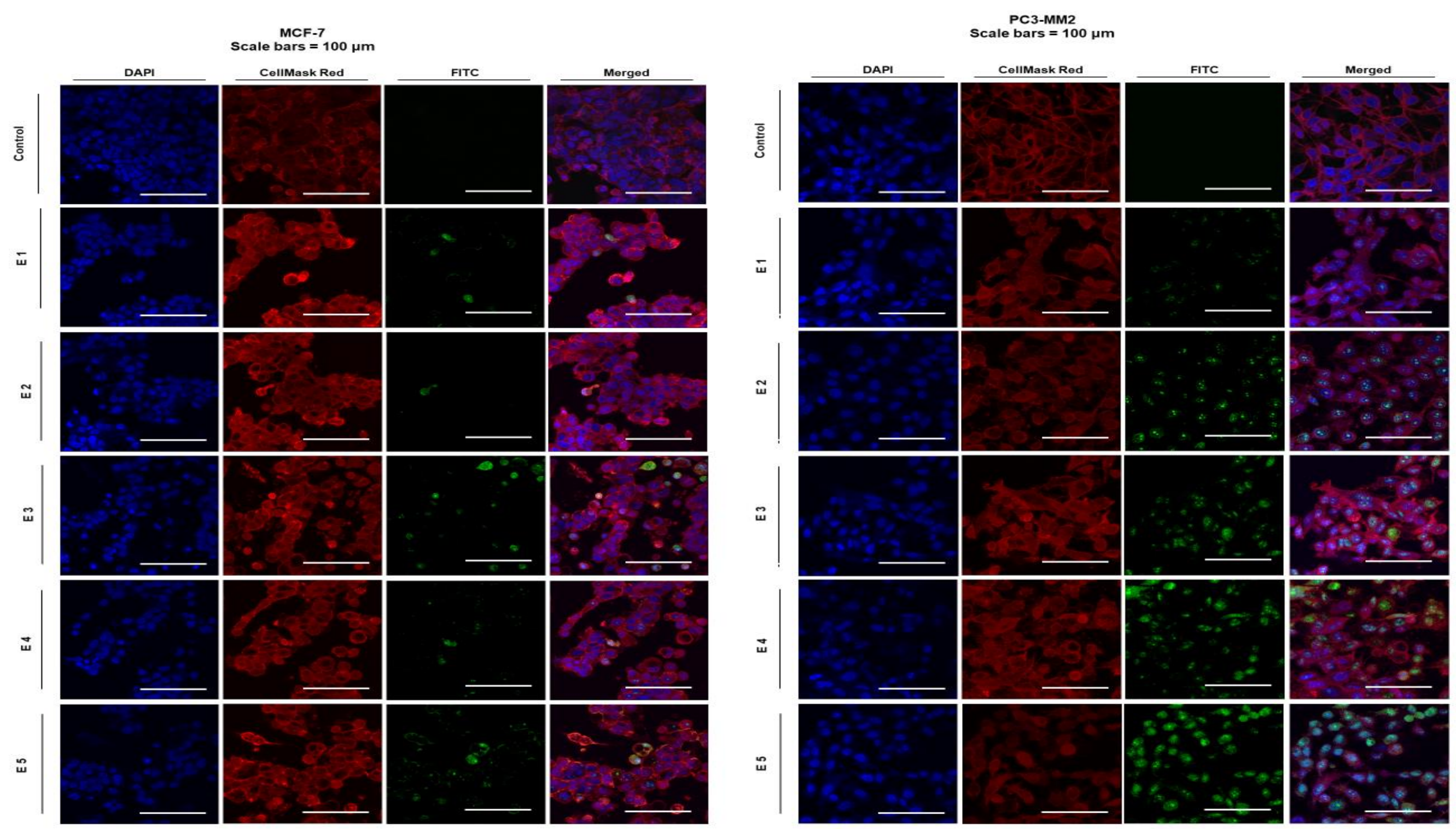

Figure 6. Confocal images of niosomal formulations uptake by MCF-7 and PC3-MM2 cells. 


\subsection{Antiproliferative studies}

The antiproliferative activity of the anticancer drugs (DOX, PXT) loaded niosomes was measured by ATP activity based luminescence assay as indicated in Figure 7 . The effect of pure drugs, empty niosomes, and niosomes containing the anticancer drugs, was evaluated on MCF7 and PC3-MM2 cell lines. The effect of the pure drugs was seen to be concentration dependent. DOX showed more antiproliferative effect on PC3-MM2 cells as compared to PXT pure drug at highest concentration levels $(500 \mu \mathrm{g} / \mathrm{ml}, \mathrm{P}<0.05)$, but in MCF-7 cell line there were no statistical difference in antiproliferative effect between the pure drugs in the highest concentration. The niosomal formulations showed time and concentration dependent type of activity.

In MCF-7 cell line the formulations containing both drugs didn't differed from formulations having either of the drug alone. But, In PC3-MM2 cells, dual drug therapy (DOX+PTX niosomes) showed better antiproliferative effect on cancerous cell lines and efficacy of the niosomal formulations as compared to either DOX alone $(\mathrm{P}<0.01)$ or PTX alone $(\mathrm{P}<0.05)$. The formulations containing paclitaxel and doxorubicin (DP1-DP5) showed improvement of antiproliferation against MCF-7 and PC3-MM2 cells. The efficacy against PC3-MM2 is much higher than MCF-7 ( $\mathrm{P}<0.01)$. It showed concentration and time dependent activity.

The in-vitro release studies showed the sustained drug release behavior of the formulations, which is also clear from the cell line antiproliferation studies. The increase in incubation time of cells would improve the effects. But, the results were in line with the results of release studies of the formulations, which was sustained drug release which is also reported in previous findings (Tahir et al., 2017; Alemi et al., 2018). 


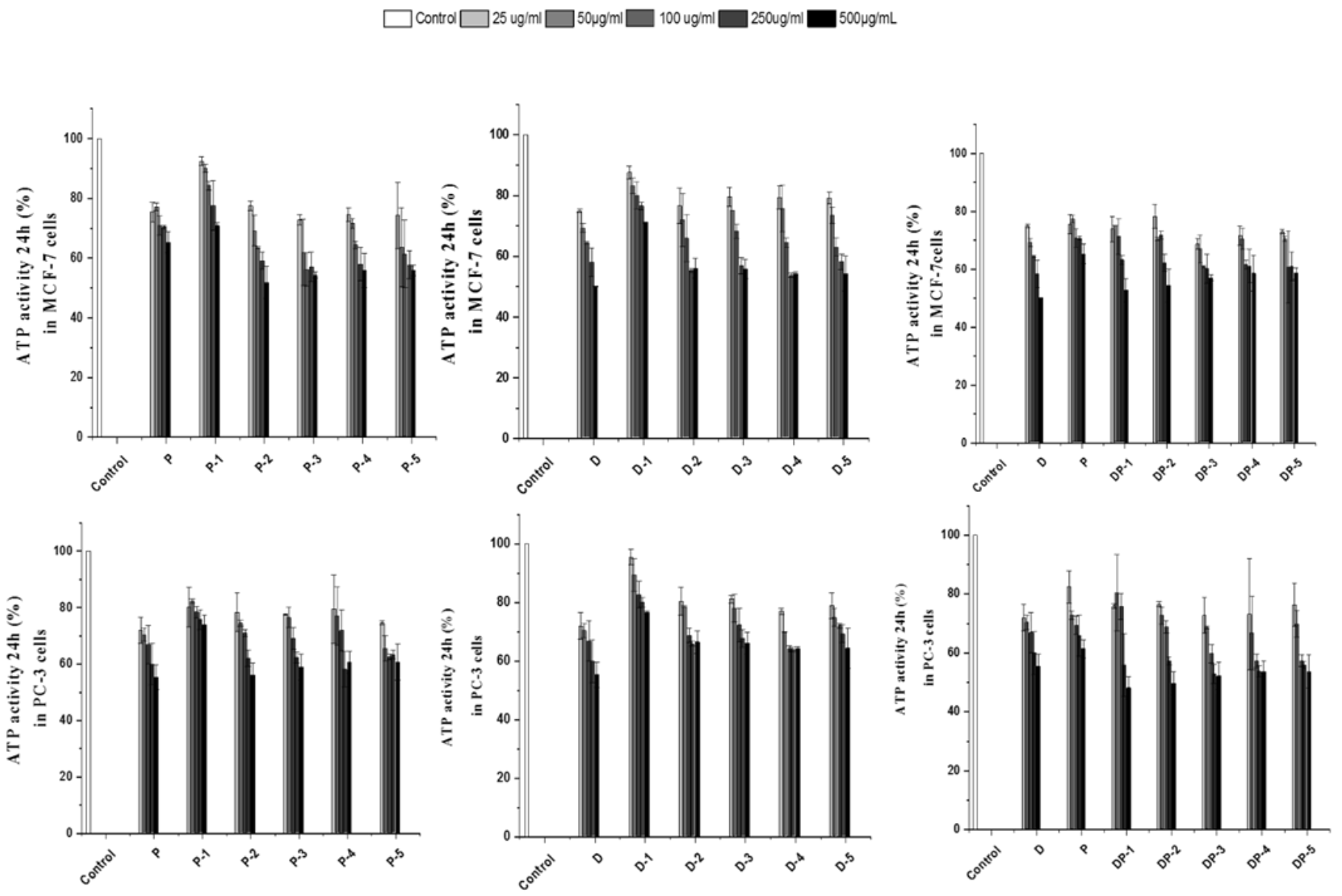

Figure 7. Antiproliferative study of different concentrations of drugs on MCF-7 and PC3-MM2 cell after $24 \mathrm{~h}$ incubation time. 


\section{Conclusion}

In the present study, two cytotoxic drugs, doxorubicin and paclitaxel, were encapsulated into niosome formulations by using an ecofriendly probe sonication method. The niosomes prepared with fixed amount of Span 60 and cholesterol, and with varying amount of Pluronic L121 and dicetylphosphate showed high entrapment efficiencies, as well as acceptable size and monodispersity levels. In-vitro characterization showed that the formulations had sustained drug release profiles, low toxicity even at high concentrations, better cell penetration and improved antiproliferative effects in time and dose dependent manner. Further, niosomes showed synergistic effect due to the presence of two anticancer drugs.

\section{Acknowledgements}

This work was supported by The International Research Support Initiative Program of Higher Education Commission of Pakistan [travel grant awarded to Mr. Daulat Haleem Khan for the research visit in University of Helsinki, Finland], the Sigrid Jusélius Foundation [grant number 4704580], and the HiLIFE Research Funds. 


\section{References}

Abdelbary, G.A., Tadros, M.I., 2013. Brain targeting of olanzapine via intranasal delivery of core-shell difunctional block copolymer mixed nanomicellar carriers: In vitro characterization, ex vivo estimation of nasal toxicity and in vivo biodistribution studies. Int. J. Pharm. 452, 300310. doi:10.1016/j.ijpharm.2013.04.084.

Ag Seleci, D., Seleci, M., Stahl, F., Scheper, T., 2017. Tumor homing and penetrating peptideconjugated niosomes as multi-drug carriers for tumor-targeted drug delivery. RSC Adv. 7 , 33378-33384. doi:10.1039/c7ra05071b.

Alemi, A., Zavar Reza, J., Haghiralsadat, F., Zarei Jaliani, H., Haghi Karamallah, M., Hosseini, S.A., Haghi Karamallah, S., 2018. Paclitaxel and curcumin coadministration in novel cationic PEGylated niosomal formulations exhibit enhanced synergistic antitumor efficacy. J. Nanobiotechnol. 16, 1-20. doi:10.1186/s12951-018-0351-4.

Al-Lazikani, B., Banerji, U., Workman, P., 2012. Combinatorial drug therapy for cancer in the post-genomic era. Nat. Biotechnol. 30, 679-692. doi:10.1038/nbt.2284.

Basiri, L., Rajabzadeh, G., Bostan, A., 2017. Physicochemical properties and release behavior of Span 60/Tween 60 niosomes as vehicle for $\alpha$-Tocopherol delivery. LWT 84, 471-478. doi:10.1016/j.lwt.2017.06.009.

Behnam, B., Rezazadehkermani, M., Ahmadzadeh, S., Mokhtarzadeh, A., Nematollahi-Mahani, S.N., Pardakhty, A., 2018. Microniosomes for concurrent doxorubicin and iron oxide nanoparticles loading; preparation, characterization and cytotoxicity studies. Artif. Cells Nanomed. Biotechnol. 46, 118-125. doi:10.1080/21691401.2017.1296850.

Chabner, B.A., Roberts, T.G., 2005. Timeline: chemotherapy and the war on cancer. Nat. Rev. Cancer 5, 65-72. doi:10.1038/nrc1529. 
Devita Jr, G.P., Young, V.T., Canellos, R.C., 1975. Combination versus single agent chemotherapy: a review of the basis for selection of drug treatment of cancer. Cancer 35, 98110. doi:10.1016/B978-0-08-023256-0.50055-3.

Di Marzio, L., Marianecci, C., Petrone, M., Rinaldi, F., Carafa, M., 2011. Novel pH-sensitive non-ionic surfactant vesicles: comparison between Tween 21 and Tween 20. Colloids Surf.B Biointerfaces 82, 18-24. doi:10.1016/j.colsurfb.2010.08.004.

Doustgani, A., 2017. Doxorubicin release from optimized electrospun polylactic acid nanofibers. J. Ind. Text. 47, 71-88. doi:10.1177/1528083716634033.

Dufes, C., Schätzlein, A.G., Tetley, L., Gray, A.I., Watson, D.G., Olivier, J.-C., Couet, W., Uchegbu, I.F., 2000. Niosomes and polymeric chitosan based vesicles bearing transferrin and glucose ligands for drug targeting. Pharm. Res. 17, 1250-1258. doi:10.1023/A:1026422915326.

Escudero, I., Geanta, R.M., Ruiz, M.O., Benito, J.M., 2014. Formulation and characterization of Tween 80/cholestherol niosomes modified with tri-n-octylmethylammonium chloride (TOMAC) for carboxylic acids entrapment. Colloids Surf. A Physicochem. Eng. Asp. 461, 167-177. doi:10.1016/J.COLSURFA.2014.07.042.

Figueiredo, P., Lintinen, K., Kiriazis, A., Hynninen, V., Liu, Z., Bauleth-Ramos, T., Rahikkala, A., Correia, A. Kohout, T., Sarmento, B., Yli-Kauhaluoma, J., Hirvonen, J., Ikkala, O., Kostiainen, M.A., Santos, H.A., 2017. In vitro evaluation of biodegradable lignin-based nanoparticles for drug delivery and enhanced antiproliferation effect in cancer cells. Biomaterials 121, 97-108. doi:10.1016/j.biomaterials.2016.12.034.

Guo, Y., Wang, L., Lv, P., Zhang, P., 2015. Transferrin-conjugated doxorubicin-loaded lipidcoated nanoparticles for the targeting and therapy of lung cancer. Oncol. Lett. 9, 1065-1072. doi:10.3892/ol.2014.2840. 
Helal, D.A., Teaima, M.H., El-Rhman, D.A., Abdel-Halim, S.A., El-Nabarawi, M., 2015. Preparation and evaluation of niosomes containing an anticellulite drug. Inven. Impact Pharma Tech. 2, 95-101.

Kanaani, L., Tabrizi, M.M., Khiyavi, A.A., 2017. Improvement the efficacy of cisplatin by niosome nanoparticles against human breast cancer cell line BT-20: an in vitro study. Asian Pacific J. Cancer Biol. 2, 25-26. doi:10.22034/APJCB.2017.2.2.25.

Khan, D.H., Bashir, S., Figueiredo, P., Santos, H.A., Khan, K.I., Peltonen, L., 2019. Process optimization of ecological probe sonication technique for production of rifampicin loaded niosomes. J. Drug Deliv. Sci. Technol. 50, 27-33. doi:10.1016/j.jddst.2019.01.012.

Khan, M.I., Madni, A., Ahmad, S., Khan, A., Rehmanand, M., Mahmood, M.A., 2015. ATRFTIR based pre and post formulation compatibility studies for the design of niosomal drug delivery system containing nonionic amphiphiles and chondroprotective drug. J. Chem. Soc. Pakistan 37, 527-535.

Khan, M.I., Madni, A., Peltonen, L., 2016. Development and in-vitro characterization of sorbitan monolaurate and poloxamer 184 based niosomes for oral delivery of diacerein. Eur. J. Pharm. Sci. 95, 88-95. doi:10.1016/j.ejps.2016.09.002.

Khan, M.I., Madni, A., Hirvonen, J., Peltonen, L., 2017. Ultrasonic processing technique as a green preparation approach for diacerein-loaded niosomes. AAPS PharmSciTech 18, 15541563. doi:10.1208/s12249-016-0622-z.

Khutale, G.V., Casey, A., 2017. Synthesis and characterization of a multifunctional golddoxorubicin nanoparticle system for $\mathrm{pH}$ triggered intracellular anticancer drug release. Eur. J. Pharm. Biopharm. 119, 372-380. doi:10.1016/j.ejpb.2017.07.009. 
Kim, J.H., Kim, Y., Bae, K.H., Park, T.G., Lee, J.H., Park, K., 2015. Tumor-targeted delivery of paclitaxel using low density lipoprotein-mimetic solid lipid nanoparticles. Mol. Pharm. 12, 1230-1241. doi:10.1021/mp500737y.

Lee, E.S., Na, K., Bae, Y.H., 2005. Super pH-sensitive multifunctional polymeric micelle. Nano Lett. 5, 325-329. doi:10.1021/n10479987.

Lee, E.S., Oh, Y.T., Seok Youn, Y., Nam, M., Park, B., Yun, J., Kim, J.H., Song, H.-T., Oh, K.T., 2011. Binary mixing of micelles using Pluronics for a nano-sized drug delivery system. Colloids Surf. B Biointerfaces 82, 190-195. doi:10.1016/j.colsurfb.2010.08.033.

Li, F.-T., Zhao, D.-S., Luo, Q.-Z., Liu, R.-H., Yin, R., 2008. Research on surface-modification of nano-TiO2 by span 60. J. Ceram. Process. Res. 9, 398-400.

Li, W.Z., Hao, X.L., Zhao, N., Han, W.X., Zhai, X.F., Zhao, Q., Wang, Y.E., Zhou, Y.Q., Cheng, Y.C., Yue, Y.H., Fu, L.N., Zhou, J.L., Wu, H.Y., Dong, C.J., 2016. Propylene glycol-embodying deformable liposomes as a novel drug delivery carrier for vaginal fibrauretine delivery applications. J. Control. Release 226, 107-114. doi:10.1016/j.jconrel.2016.02.024.

Liu, H., Tu, L., Zhou, Y., Dang, Z., Wang, L., Du, J., Feng, J., Hu, K., 2017. Improved bioavailability and antitumor effect of docetaxel by TPGS modified proniosomes: in vitro and in vivo evaluations. Sci. Rep. 7, 43372. doi:10.1038/srep43372.

Maestrelli, F., Mura, P., González-Rodríguez, M.L., Cózar-Bernal, M.J., Rabasco, A.M., Di Cesare Mannelli, L., Ghelardini, C., 2017. Calcium alginate microspheres containing metformin hydrochloride niosomes and chitosomes aimed for oral therapy of type 2 diabetes mellitus. Int. J. Pharm. 530, 430-439. doi:10.1016/j.ijpharm.2017.07.083. 
Mahale, N.B., Thakkar, P.D., Mali, R.G., Walunj, D.R., Chaudhari, S.R., 2012. Niosomes: novel sustained release nonionic stable vesicular systems - an overview. Adv. Colloid Interface Sci. 183-184, 46-54. doi:10.1016/j.cis.2012.08.002.

Majeed, M.I., Lu, Q., Yan, W., Li, Z., Hussain, I., Tahir, N., Tremel, M., Tan, W., Tan, B., 2013. Highly water-soluble magnetic iron oxide (Fe3O4) nanoparticles for drug delivery: enhanced in vitro therapeutic efficacy of doxorubicin and MION conjugates. J. Mater. Chem. B 1, 28742884. doi:10.1039/c3tb20322k.

Manconi, M., Sinico, C., Valenti, D., Loy, G., Fadda, A.M., 2002. Niosomes as carriers for tretinoin. I. Preparation and properties. Int. J. Pharm. 234, 237-248. doi:10.1016/S03785173(01)00971-1.

Martins, K.F., Messias, A.D., Leite, F.L., Duek, E.A.R., 2014. Preparation and characterization of paclitaxel-loaded PLDLA microspheres. Mater. Res. 17, 650-656. doi:10.1590/S151614392014005000028.

Moghassemi, S., Hadjizadeh, A., 2014. Nano-niosomes as nanoscale drug delivery systems: an illustrated review, J. Control. Release. 185, 22-36. doi:10.1016/j.jconrel.2014.04.015.

Nasseri, B., 2005. Effect of cholesterol and temperature on the elastic properties of niosomal membranes. Int. J. Pharm. 300, 95-101. doi:10.1016/j.ijpharm.2005.05.009.

Newman, M.-J., Balusubramanian, M., Todd, C.W., 1998. Development of adjuvant-active nonionic block copolymers. Adv. Drug Deliv. Rev. 32, 199-223. doi:10.1016/S0169409X(98)00011-8.

Pawar, S., Shevalkar, G., Vavia, P., 2016. Glucosamine-anchored doxorubicin-loaded targeted nano-niosomes: pharmacokinetic, toxicity and pharmacodynamic evaluation. J. Drug Target. 24, 730-743. doi:10.3109/1061186X.2016.1154560. 
Pawar, S., Vavia, P., 2016. Glucosamine anchored cancer targeted nano-vesicular drug delivery system of doxorubicin. J. Drug Target. 24, 68-79. doi:10.3109/1061186X.2015.1055572.

Ravalika, V., Sailaja, A.K., 2017. Formulation and evaluation of etoricoxib niosomes by thin film hydration technique and ether injection method. Nano Biomed. Eng. 9, 242-248. doi:10.5101/nbe.v9i3.p242-248.

Sayed, E., Karavasili, C., Ruparelia, K., Haj-Ahmad, R., Charalambopoulou, G., Steriotis, T., Giasafaki, D., Cox, P., Singh, N., Giassafaki, L.-P.N., Mpenekou, A., Markopoulou, C.K., Vizirianakis, I.S., Chang, M.W., Fatouros, D.G., Ahmad, Z., 2018. Electrosprayed mesoporous particles for improved aqueous solubility of a poorly water soluble anticancer agent: in vitro and ex vivo evaluation. J. Control. Release 278, 142-155. doi:10.1016/J.JCONREL.2018.03.031.

Shahbazi, M., Almeida, P.V., Mäkilä, E., Correia, A., Ferreira, M.P., Kaasalainen, M., Salonen, J., Hirvonen, J., Santos, H.A., 2014. Functionalized porous silicon nanoparticles for enhanced stability and cellular internalization. Macromol. Rapid Commun. 35, 624-629.

Sharma, V., Anandhakumar, S., Sasidharan, M., 2015. Self-degrading niosomes for encapsulation of hydrophilic and hydrophobic drugs: an efficient carrier for cancer multi-drug delivery. Mater. Sci. Eng. C. 56, 393-400. doi:10.1016/j.msec.2015.06.049.

Sharma, P.K., Saxena, P., Jaswanth, A., Chalamaiah, M., Tekade, K.R., Balasubramaniam, A., 2016. Novel encapsulation of lycopene in niosomes and assessment of its anticancer activity. J. Bioequiv. Availab. 8, 224-232. doi:10.4172/jbb.1000300.

Shi, K., Zhou, J., Zhang, Q., Gao, H., Liu, Y., Zong, T., He, Q, 2015. Arginine-glycine-aspartic acid-modified lipid-polymer hybrid nanoparticles for docetaxel delivery in glioblastoma multiforme. J. Biomed. Nanotechnol. 11, 382-391. doi:10.1166/jbn.2015.1965. 
Somjid, S., Krongsuk, S., Johns, J.R., 2018. Cholesterol concentration effect on the bilayer properties and phase formation of niosome bilayers: a molecular dynamics simulation study. J. Mol. Liq. 256, 591-598. doi:10.1016/j.molliq.2018.02.077.

Tahir, N., Madni, A., Balasubramanian, V., Rehman, M., Correia, A., Kashif, P.M., Mäkilä, E., Salonen, J., Santos, H.A., 2017. Development and optimization of methotrexate-loaded lipidpolymer hybrid nanoparticles for controlled drug delivery applications. Int. J. Pharm. 533, 156168. doi:10.1016/j.ijpharm.2017.09.061.

Tavano, L., Aiello, R., Ioele, G., Picci, N., Muzzalupo, R., 2014. Niosomes from glucuronic acidbased surfactant as new carriers for cancer therapy: Preparation, characterization and biological properties. Colloids Surf. B Biointerfaces 118, 7-13. doi:10.1016/j.colsurfb.2014.03.016.

Teixeira, M.C., Carbone, C., Souto, E.B., 2017. Beyond liposomes: recent advances on lipid based nanostructures for poorly soluble/poorly permeable drug delivery. Prog. Lipid Res. 68, 111. doi:10.1016/J.PLIPRES.2017.07.001.

Yang, T., De Cui, F., Choi, M.K., Cho, J.W., Chung, S.J., Shim, C.K., Kim, D.D., 2007 a. Enhanced solubility and stability of PEGylated liposomal paclitaxel: in vitro and in vivo evaluation. Int. J. Pharm. 338, 317-326. doi:10.1016/j.ijpharm.2007.02.011.

Yang, T.F., Chen, C.N., Chen, M.C., Lai, C.H., Liang, H.F., Sung, H.W., 2007b. Shellcrosslinked Pluronic L121 micelles as a drug delivery vehicle. Biomaterials 28, 725-734. doi:10.1016/j.biomaterials.2006.09.035.

Yang, M., Ding, H., Zhu, Y., Ge, Y., Li, L., 2019. Co-delivery of paclitaxel and doxorubicin using mixed micelles based on the redox sensitive prodrugs. Colloids Surf. B Biointerfaces 175, 126-135. doi:10.1016/j.colsurfb.2018.11.086. 
Yingchoncharoen, P., Kalinowski, D.S., Richardson, D.R., 2016. Lipid-based drug delivery systems in cancer therapy: what is available and what is yet to come. Pharmacol. Rev. 68, 701787. doi:10.1124/pr.115.012070.

Zheng, M., Gong, P., Zheng, C., Zhao, P., Luo, Z., Ma, Y., Cai, L., 2015. Lipid-polymer nanoparticles for folate-receptor targeting delivery of doxorubicin. J. Nanosci. Nanotechnol. 15, 4792-4798. doi:10.1166/jnn.2015.9604. 